\title{
Determinants of polydipsia in rats: II. DRL extinction'
}

\author{
Evalyn F. Segal and Sam A. Deadwyler \\ SAN DIEGO STATE COLLEGE
}

\begin{abstract}
Three rats were trained on DRL 30 sec. for food. All developed polydipsia. Two DRL extinction sessions were run, one with the dispenser click following each correctly-spaced response, one without it. Drinking continued through extinction sessions, at lower frequency. Two of the rats drank more in the extinction session with the click than in the one without it.

\section{Problem}

Stein (1964) has argued that the excessive drinking by rats on dry-food reinforcement schedules is due solely to thirst. Although thirst no doubt is one factor, it does not follow that other factors play no role. This is the second in a series of papers in which we hope to show the role of other variables. In the first paper (Segal \& Oden, 1965) we showed that even food-reinforced licking is disrupted by emptying the water bottle, refuting Stein's (1964) assumption that such disruption proves the control of thirst alone over licking.

Here we will show that (1) polydipsia induced by a DRL continues even in sessions that include no food, and (2) more drinking occurs in extinction sessions that include operation of the empty food dispenser than in sessions that do not. We conclude that part of the control of polydipsia comes from the discriminative properties of the dispenser click, and part, perhaps, from more subtle contingencies of interval schedules.
\end{abstract}

\section{Method}

Three adult, male, albino rats, maintained at $80 \%$ of ad lib weight, were trained for several months on a DRL 30 sec. schedule of bar pressing, during which we observed the effect of changing the palatability of the drinking solution and administering amphetamine. Re-

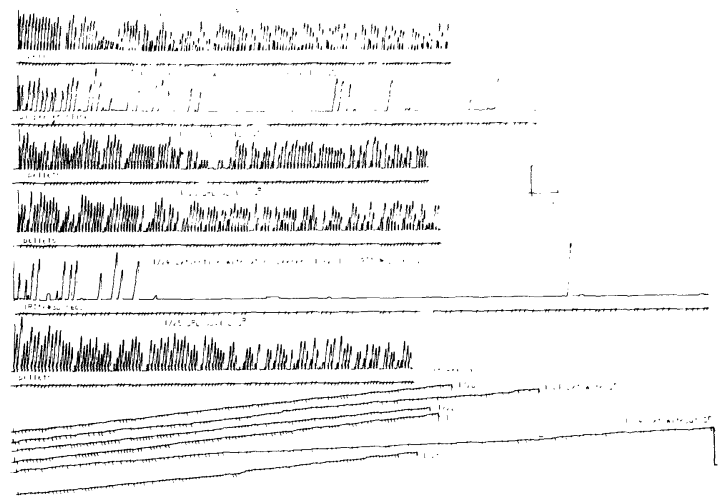

Fig. 1. Bar pressing and drinking in A-3. inforcers were $45 \mathrm{mg}$ Noyes peanut pellets. The conditioning chamber had a bar, a food cup, and a water nozzle, and was housed in a sound-insulated chamber in a sound-resistant room containing white masking noise.

This experiment consisted of 1 control day, 1 extinction day during which responses spaced $\geq 30 \mathrm{sec}$. apart operated the empty food dispenser, 2 more control days, 1 extinction session during which presses had no experimental effect, and 1 more control day. Control sessions were 100 pellets long. The first extinction session was 100 dispenser clicks long. Extinction session 2 lasted for 60 IRTs $\geq 30 \mathrm{sec}$. in BC-2, 80 in $\mathrm{A}-2$, and 100 in $\mathrm{A}-3$; the session was $\leq 2 \mathrm{hr}$. long.

\section{Result:}

Figures 1 \& 2 show drinkingand bar pressing of two of the Ss. The third $\mathrm{S}, \mathrm{A}-2$, behaved similarly to A-3. (Every bar press, reinforced or not, reset the drinking pen to baseline.)

All Ss drank continually through control sessions. BC-2 rarely drank except following a pellet. The other Ss sometimes drank following non-reinforced as well as reinforced presses. All Ss drank through the early part of extinction session 1; BC-2 drank the least. There was little drinking in the later part of the session. All Ss drank in the early part of extinction session 2; A-2 and A-3 stopped drinking sooner in this than in extinction session 1 .

Figure 3 is a summary. The top row shows percentage of reinforced (solid line) and non-reinforced (dashed line) presses that were followed within a few sec. by drinking. (In extinction session 1 reinforcement was the dispenser click. Extinction session 2 included no rein-



Fig. 2. Bar pressing and drinking in $\mathrm{BC}-2$. 


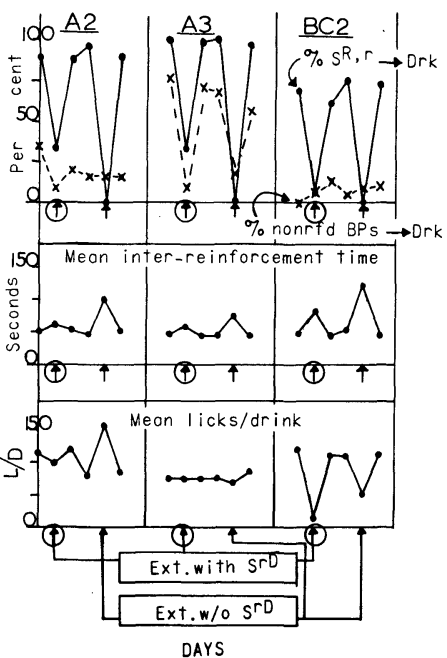

DRL 30:Extinction with or w/o dispenser click(SrD)

Fig. 3. Top: per cent of reinforced and non-reinforced bar presses followed by drinks. Middle: mean inter-reinforcement time. Bottom: mean licks per drink.

forcement.) All Ss were more inclined to drink following reinforced than non-reinforced responses, and all were more inclined to drink following food than dispenser clicks. The dashed curves for all sessions were fairly stable for A-2 and BC-2; only A-3 showed a covariation between percentage of drinks following reinforced and non-reinforced presses.

The second row shows the mean inter-reinforcement time. (For extinction session 2, read as the mean time between occurrences of IRTs $\geq 30 \mathrm{sec}$.) Only in BC-2 did extinction session 1 increase mean inter-reinforcement time appreciably. The other Ss maintained good bar-press spacing. Extinction session 2 increased inter-S ${ }^{R}$ time in all Ss. This disruption of timing was greatest in BC-2.

The third row shows the mean number of licks in a drink. It was stable over all sessions, for A-3; increased in extinction session 2, for A-2; and fell in both extinction sessions, for $\mathrm{BC}-2$.

\section{Diseussion}

It appears to us that A-2 and A-3 drank for reasons additional to thirst, while BC-2 drank for thirst only. BC-2 rarely drank except following pellets; it showed the greatest disruption in DRL behavior in extinction sessions. Clearly, drinking did not mediate $\mathrm{BC}-2$ 's response spacing, except in the trivial sense that when it did drink, time passed, and so presses were more likely to be reinforced.

A-2 and A-3 drank frequently following non-reinforced presses, and frequently following clicks in extinction session 1. Part of the control of drinking came from food, for the Ss drank less when food was omitted. But part came from the discriminative properties of the click, and part may have come from the reinforcement contingencies of the DRL. These Ss seem to have learned to mediate their response spacing by drinking. So long as they continued to drink (extinction session 1) they continued to space their bar presses accurately. Only when they stopped drinking (extinction session 2) did response spacing worsen. As we have earlier said (Segal \& Deadwyler, 1964), the phrase mediating behavior ought to be used only where the behavior can be shown to supply the unique cue for the spaced response. We regard the difference between mean inter $-S^{R}$ time in extinction sessions 1 and 2 as suggestive, although not definitive. So long as the click, and drinking, continued, so long did DRL performance remain stable. It seems likely that drinking supplied the timing cue. However, an alternative explanation that cannot be ruled out is that the click maintained spaced responding in its role as conditioned reinforcer, and that drinking was only incidental to timing.

Referenees

SEGAL, EVALYN F., \& DEADWYLER, S. A. Amphetamine differentially affects spaced bar pressing and collateral water drinking. Psychon. Sci., 1964, 1, 349-350.

SEGAL, EVALYN F., \& ODEN, D. L. Determinants of polydipsia in rats: A reply to Stein. I. Emptying the water bottle. Psychon. Sci., 1965, 201-202.

STEIN, L. Excessive drinking in the rat: Superstition or thirst? J. comp. physiol. Psychol., 1964, 58, 237-242.

Note

1. Supported by NSF GB 1605 and NIMH 8505. 